\title{
La problemática socionatural: cómo la migración reproduce las desigualdades en la era del cambio climático
}

\author{
The socio-natural problematic: how migration reproduces \\ inequalities in the era of climate change
}

Thomas Faist*

ISSN IMPRESO 1870-7599 | ISSN RED CÓMPUTO 2448-7783 | 11-29

\begin{abstract}
Resumen. La migración transfronteriza es una de las diversas maneras en que las personas se han adaptado al lento y al rápido advenimiento de los cambios medioambientales del Antropoceno, era en que las actividades del hombre han producido consecuencias globales relevantes hacia los ecosistemas del mundo. A fin de explorar las desigualdades se requieren estudios sistemáticos acerca de cómo la migración surge a partir de complejas interacciones entre los procesos sociales (políticos, económicos, culturales) y medioambientales. Hasta ahora, dos generaciones de académicos se han referido al debate del cambio climático y la migración. La primera teorizó la migración como una respuesta mecánica al cambio climático; mientras que la segunda conceptualizó la migración vinculada al cambio climático como una adaptación en las relaciones y en la seguridad humana, al ubicar en el centro de la cuestión las acciones del hombre. El énfasis en el «migrante resiliente» ha negado los efectos del cambio climático en las categorías de la clasificación de personas según las desigualdades sociales. Este estudio analiza la evolución de los conceptos en los debates acerca del nexo migración y cambio climático y algunos mecanismos sociales de (re)producción de las desigualdades.
\end{abstract}

Palabras clave: cambio climático, seguridad humana, migrante resiliente, inequidad social.

\begin{abstract}
Cross-border migration is one of the many ways in which people have adapted to both the slow and sudden advance of environmental changes in the Anthropocene epoch, in which the activities of human beings have produced global consequences for the world's ecosystems. This paper aims to explore the inequalities that require systematic study with regard to how migration increases as a result of the complex interactions between social (political, economic, cultural) and environmental processes. To date, two generations of researchers have engaged the climate change debate as it relates to migration. The first theorized migration as a mechanical response to climate change; while the second conceptualized migration as being tied to climate change as an adaptation in the relations governing human security, situating at its core the question of human beings' actions. The emphasis on the "resilient migrant» has omitted the effects of climate change on the categories of classification of people according to social inequalities. This paper analyzes the evolution of those concepts in the debates around the migration-climate change nexus and some of the social mechanisms of inequality reproduction.
\end{abstract}

Keywords: climate change, human security, resilient migrant, social inequalities.

\footnotetext{
${ }^{*}$ Profesor de Estudios Transnacionales, Desarrollo y Migración de la Universidad de Bielefeld, Alemania. Traducido del inglés por Jorge Miguel Veizaga Rosales y Georgia Aralú González Pérez.
} 



\section{Cambio climático y migración en el Antropoceno}

El debate sobre migración y cambio climático ha sido alentado y sostenido por los reiterados y terribles escenarios relativos al número de personas que supuestamente serían desplazadas en las próximas décadas. Las cifras oscilan entre 200 y 700 millones de «refugiados climáticos» para el año 2050 (cfr. Myers y Kent, 1995; Christian Aid, 2007). El cambio climático «se atribuye directa o indirectamente a la actividad humana que altera la composición de la atmósfera global y en la cual se observa la variabilidad natural climática a lo largo de periodos comparables» (UNFCCC, 1992:3).

Desde finales de la década de 1980, los Reportes Evaluativos del Grupo Intergubernamental de Expertos sobre el Cambio Climático (IPCC por sus siglas en inglés) han sido parte del constructo científico del consenso que se ha mantenido relativamente estable con respecto a un factor clave: la sensibilidad del clima de acuerdo con la duplicación del $\mathrm{CO}_{2}$ atmosférico, expresada en el incremento previsto de la temperatura media global. Existe además evidencia que sugiere, por un lado, una correlación positiva entre el volumen de los flujos migratorios de 172 países y, por el otro, los indicadores de pesca excesiva, desertización, escasez de agua, salinización del suelo, deforestación, contaminación del aire, erosión del suelo y consecuente contaminación (por ejemplo, Faist y Schade, 2013). De manera que los efectos negativos del cambio climático son valorados como un caso especial de la degradación medioambiental. Esto último implica también procesos que no se relacionan ni casual ni directamente al calentamiento global, por ejemplo el impacto de los proyectos de desarrollo (cfr. construcción de represas y proyectos de desarrollo).

El pensamiento simplista que subyace a los desalentadores escenarios acerca del dramático incremento de refugiados climáticos es que las emisiones de Gases de Efecto Invernadero (GEI) en particular y la degradación medioambiental en general aluden a que las personas están obligadas a responder de modo directo a las consecuencias del cambio climático, tal es el caso de la elevación del nivel del mar, hecho que propicia migrar hacia áreas de mayor altitud. Aunque al parecer la naturaleza y la sociedad/cultura pueden separarse claramente; es una dicotomía falsa. Sería mejor concebirla como un vínculo. La división entre naturalezasociedad y naturaleza-cultura asume una respuesta mecánica unilineal, insostenible por dos razones. Primera, los seres humanos han alterado el clima en el que 
vivimos, de ahí que el químico Paul Crutzen en los 1990 popularizó el término Antropoceno, el cual sostiene que los humanos se han convertido en una fuerza geológica. Segunda, los riesgos asociados con el cambio climático se experimentan de distinta manera por diversos grupos de individuos.

En resumen, las vulnerabilidades son diferenciadas. Ello es comprensible si se observan las desigualdades sociales involucradas en la dinámica de la degradación medioambiental y en la movilidad de los afectados. Lo anterior puede verse como una suerte de relación transversal: a pesar de que las emisiones de GEI no pueden ser detenidas por las patrullas fronterizas, las consecuencias en las desigualdades sociales son muy irregulares. Los sectores más pobres de la población mundial, es decir, aquellos que menos han contribuido al calentamiento global, son los más golpeados (Human Development Report, 2007). Aun así, gracias a décadas de investigación sobre migración internacional, se sabe que los estratos más pobres de las sociedades son los más proclives a participar en la migración transfronteriza (Ahmed, 1997). En ese sentido, la relación entre la migración inducida por el cambio climático y las desigualdades sociales asociadas deben complementarse desde una perspectiva en correspondencia con factores socionaturales inherentes. Dicho enfoque enfatiza de igual modo que el cambio climático no sólo se refiere a la previsión científicamente determinada de los peligros, sino que además debe integrar aspectos sociales y naturales. Por ende, las interrogantes empíricas fundamentales son las siguientes: ¿̇de qué manera impactan las desigualdades sociales del cambio climático en la migración y cómo ésta (re)produce tales desigualdades?, żqué mecanismos operan?

El Antropoceno se caracteriza tanto por un aspecto social —el conflicto entre el capital y el trabajo en el siglo XIX — como por un aspecto socionatural. En otras palabras, no nos enfrentamos únicamente a las dinámicas y a las consecuencias de la explotación de humanos por humanos, pues este mecanismo básico se correlaciona con la explotación de la naturaleza por los seres humanos - y las consecuencias que esa explotación entraña en las relaciones sociales en un sentido amplio - sino también a las implicaciones migratorias y las desigualdades en el contexto del cambio climático presentes en las investigaciones. Los efectos del cambio climático han producido muy diversos procesos: existe la migración que deriva de la lenta degradación y aquella del cambio rápido. Un ejemplo del cambio lento se observa en varios de los Estados Isla del Pacífico en el transcurso de la década pasada (cfr. Shen y Gemenne, 2011) y 
desastres masivos, tal es el caso de las inundaciones de distintas ciudades en los deltas de los ríos, por ejemplo en Bangladesh (Etzold et al., 2016).

Los efectos del cambio climático de inicio lento podrían ser más drásticos en el futuro, de ahí que sea imprescindible tomar en cuenta la paradoja de la degradación. ${ }^{1}$ Ésta consiste en que las secuelas negativas del cambio climático no son visibles de inmediato en la vida diaria. Así, el argumento indica que la mayoría de la gente no hará nada al respecto hasta que las consecuencias sean devastadoras. No obstante, en ese momento podría ser demasiado tarde, tanto para mitigar el cambio climático como para adaptarse de manera creativa. Podríamos comparar semejante situación con la que enfrenta un fumador. Los fumadores normalmente son conscientes de que su hábito es peligroso, incluso la mayor parte del daño se produce antes de que comiencen a experimentar reacciones negativas en su salud, de modo que dejan de fumar hasta que ya es demasiado tarde. La paradoja de la degradación posee importantes implicaciones en los estudios de la migración en el sentido de que no debemos esperar que el cambio climático se genere seguido por un desplazamiento inmediato. Con todo y eso, se ha hecho hincapié en los múltiples casos de cambio climático de inicio rápido (Black, Kniveton y Schmidt-Verkerk, 2013).

Ya sea que se considere un cambio climático de inicio lento o de inicio rápido, los estudios migratorios son óptimos cuando se analizan las dinámicas internas del movimiento transfronterizo y se aplican conceptos que comprenden las dinámicas endógenas (autogeneradas): causación acumulativa y pobreza relativa (véase, por ejemplo, Massey, 1990). Sin duda esto debe continuar. Debe considerarse más que antes la forma en que los factores exógenos - como el cambio climático- se convierten en endógenos dentro de los procesos de movilidad, es decir, tomar en cuenta cómo las personas y las organizaciones tratan de hacer frente a la transformación de su entorno.

Bajo esta perspectiva es necesario partir de un antiguo y valioso supuesto acerca del «excepcionalismo» del ser humano, en especial en la sociología. Durante gran parte del siglo XX, los sociólogos insistieron en la relación dual que mantienen los seres humanos con el medioambiente, al estar sujetos a límites biológicos y físicos, aunque nunca negaron su capacidad única de cultura y de comunicación simbólica. En realidad, ese es el trasfondo en contra de las suposiciones sociológicas acerca de los hechos sociales. No sorprende que una de sus

${ }^{1}$ También se la conoce como la «Paradoja de Giddens» (Giddens, 2009), ya que Anthony Giddens aplicó esta vieja idea al cambio climático. 
premisas centrales sea que un hecho social siempre se basará en otro hecho social en oposición al hecho psicológico. Lo anterior tiene su origen en una de las figuras fundadoras de la sociología quien sostiene que la «realidad objetiva de los hechos sociales» (Durkheim, 1964) supera a todos los esfuerzos por incorporar factores naturales. Ello impide la discusión de hechos que no sean puramente sociales, por ende, rechaza o al menos subestima los hechos biológicos y físicos, incluso realiza ciertas objeciones a la investigación sobre la confluencia de los factores biológicos, físicos y sociales. Consecuentemente, en la actualidad existe una creencia generalizada de que el entorno biofísico podría ser ignorado. Dicho fundamento también se vincula con las creencias acerca de la naturaleza excepcional de la especie humana. Lo cierto es que, tal como el cambio climático lo muestra, los seres humanos en definitiva no estamos exentos de las limitaciones ecológicas quizá porque manipulamos los principios naturales. Es evidente que debemos rechazar ese «excepcionalismo» (Dunlap y Catton, 1979) en los estudios migratorios e incluir lo que podría denominarse hechos socionaturales.

Es prioritario ejecutar acciones para trascender la perspectiva del «excepcionalismo». Primero, debe observarse la mutua interacción entre la naturaleza y la sociedad y la cultura. Estos enfoques se hallan de manera implícita en los textos de Karl Marx (1981), los cuales abordaron la transformación mutua de la naturaleza y la sociedad en el proceso del capitalismo, además avizoró la brutal explotación de la naturaleza por el hombre, que resultó en una «fisura metabólica» (Foster, 2000). Sin embargo, esta conceptualización todavía se refiere a la naturaleza y a la sociedad como dos entidades diferentes. Frente a ello existe una segunda tarea. Si es plausible asumir que la naturaleza y la sociedad se complementan una a otra, se tendrían que pensar como un nexo. Por ejemplo, los seres humanos son parte de su entorno socionatural al respirar aire (y gérmenes) que a su vez permite la vida y el cambio, de igual modo determina su posición en el tiempo y el espacio.

En ese contexto, la segunda parte del análisis se concentra en la manera en que las dos generaciones previas han desarrollado el debate del cambio climático y la migración. El argumento es que se requiere de una tercera generación que preste más atención al modo en que las respuestas al cambio climático, incluyendo la migración (como adaptación), participan en la reproducción de la estratificación social existente. La tercera parte detallará la relación entre la migración y las desigualdades como una cuestión socionatural en el contexto del cambio climático. 


\section{Tres generaciones en el debate del cambio climático-migración}

El vínculo entre naturaleza y sociedad/cultura bajo la forma de la relación entre el cambio climático y la migración ha atravesado hasta ahora dos generaciones enteras de académicos, pero se requiere de una tercera generación que se sustente en los argumentos de la segunda. En los 1980 y hasta principios de la década de 2000, la primera generación de académicos que estudiaban el vínculo entre migración y cambio climático se concentró en la idea del refugiado medioambiental, vulnerable tanto al lento como al rápido advenimiento de la presión del clima. La definición más difundida del refugiado medioambiental es aquella que se refiere a «las personas que han sido forzadas a dejar su hábitat tradicional, temporal o permanentemente, debido a un claro desorden medioambiental (natural y desencadenado por el hombre) que ha amenazado y afectado gravemente su calidad de vida» (El-Hinnawi, 1985).

En términos de una perspectiva alarmista y muy maximalista dicha concepción se adecua bastante bien, pues las nocivas consecuencias del cambio climático amenazan los medios de vida, la seguridad del Estado y deriva en conflictos armados, los cuales desencadenan mayor migración. De modo que los escenarios más comunes predecían que los cambios medioambientales y los desastres obligarían a millones de personas a dejar sus hogares (cfr. Myers y Kent, 1995). Siguiendo la línea de la argumentación, la presión medioambiental conduce a una mayor competencia por la tierra, la invasión de áreas ecológicamente frágiles y el empobrecimiento. Paralelamente, esos eventos provocan conflictos políticos y étnicos que precipitan la violencia y la guerra que con frecuencia son las causas inmediatas del escape de la población. En consecuencia, millones de refugiados deben salir de sus países, por lo que se espera que pidan asilo en países desarrollados, o que ingresen a ellos como migrantes indocumentados. Los que logran escapar a países vecinos son considerados una amenaza que atenta contra la cohesión social y contra la identidad nacional de la población nativa. La mayoría de estos motivos es de "sentido común»; a medida que se eleva el nivel del mar o que los bosques desaparecen, el individuo tendrá que moverse.

No obstante, hay abundante evidencia de que la secuencia causal que va de la degradación al conflicto y a la migración podría ser muy sencilla. De inmediato se planteó una crítica obvia: rara vez el deterioro de las condiciones climáticas 
es la única causa de la emigración. Por lo regular, la migración es una práctica ocasionada por múltiples razones, en este caso, la interacción de las condiciones naturales y a menudo la percepción de que las condiciones económicas y políticas son más propicias para conseguir oportunidades de vida en el extranjero. En otros términos, la migración forzada siempre tiene múltiples causas. Incluso en una situación extrema como en la de Bangladesh, ejemplo que muestra la esencia del desplazamiento medioambiental, es posible encontrar una compleja causalidad para el empobrecimiento y la migración, misma que incluye patrones de propiedad de la tierra, divisiones étnicas y principalmente proyectos de desarrollo económico. Los estudiosos de la migración se percataron de que los factores exógenos, a menos que sean de carácter extraordinario (inundaciones o erupciones de volcán) rara vez derivan en una respuesta migratoria masiva, directa y mecánica, aun en Estados Isla del Pacífico, donde el peligro de inundación es permanente en la visión de sus habitantes. Además del cambio climático, otras causas que funcionan como importantes predictores de la migración son las oportunidades económicas en el extranjero (Fornalé, Guélat y Piguet, 2016).

Pese a todo, la primera generación del debate fue crucial en la investigación, puesto que resaltó el evidente vacío en la teoría de la migración. En resumen, fue la investigación medioambiental la que dramatizó el vínculo y sugirió un número muy elevado de migrantes potenciales, mientras que los estudiosos de la migración enfatizaron en la ubicuidad de la movilidad y en la observación de que la migración inducida por el cambio lento del clima se ajustaba a los patrones de migración ya determinados. Los flujos establecidos de migración internacional no representan más de 3 por ciento de la población mundial. Si bien las teorías de migración son capaces de explicar el lento inicio del cambio y la migración, no ofrecen respuestas para el rápido inicio del cambio, hecho que denota una gran ausencia en las teorías de migración.

El debate en la primera generación de académicos se sustentaba en la premisa de que la política mitigaría el cambio climático. A medida que en el nuevo milenio era cada vez más notorio que el calentamiento global continuaría aun cuando se tomaran medidas radicales de mitigación, el discurso respecto al vínculo también comenzó a cambiar de forma gradual. En la segunda generación, la adaptación y la protección de la seguridad humana ocuparon la agenda de investigación y política. Concerniente a la adaptación, las personas debían ajustarse a condiciones climáticas y medioambientales cambiantes a fin de moderar los efectos dañinos del cambio climático. Según el 
Panel Internacional sobre el Cambio Climático (IPCC), un grupo de expertos que documenta y explica el cambio climático, es el «proceso de ajuste al clima actual o esperado y a sus efectos. En los sistemas humanos, la adaptación busca moderar los daños o aprovechar las oportunidades benéficas» (IPCC, 2014:5).

Las propuestas para la adaptación tienen dos versiones: adaptación in situ, como control de inundaciones y ajuste de cosechas o migración interna e internacional, incluyendo la relocalización. Tienen en común que no son meras respuestas y ajustes mecánicos al cambio climático, en todo caso es la intervención activa de los humanos lo que se ubica en el centro de la adaptación (cfr. Simonet, 2010). Para legitimar esta perspectiva cambiante, las teorías migratorias empezaron a desempeñar una función cada vez más importante en la investigación sobre el cambio climático y en el diseño de políticas; aunque todavía en un nivel muy bajo en comparación con el impacto de la investigación y el activismo medioambiental, que continúan indignados por las consecuencias del cambio climático. Complementariamente, los reportes del IPCC muestran que la migración se ha vuelto más significativa cuando se discuten las posibles respuestas.

Dentro de esta narrativa de adaptación emergente y en consolidación destacan los enfoques de la nueva economía de la migración laboral (NELM, por sus siglas en inglés) y de los medios de vida. Ambos enfoques sostienen que los migrantes (potenciales) asumen el riesgo ejerciendo su representación, de ahí que en los estudios migratorios se empleaba la premisa de que la movilidad espacial además de reactiva es proactiva. La NELM describe las decisiones migratorias como una respuesta a la imposibilidad de conseguir seguro formal o como una manera de diversificar el ingreso a través de la migración como un acuerdo informal de préstamo (Poirine, 1997). Por su parte, desde el enfoque de los medios de vida, que observa desigualdades en la disponibilidad de recursos y en su expansión, y de nuevo la migración, es una manera de diversificar las fuentes de ingreso mediante las remesas (Ellis, 2000).

Esta segunda generación de académicos se caracterizó también por otro cambio: la seguridad no se consideró únicamente como seguridad del Estado, sino como seguridad humana, en contraposición a la situación previa. La seguridad humana se define como «una condición que existe cuando el núcleo vital de la vida humana está protegido y cuando las personas poseen la libertad y la capacidad de vivir con dignidad» (IPCC, 2014:759). La seguridad humana se empleaba junto con otro término de moda: resiliencia. El migrante resiliente surge 
como la figura característica de aquel que se adapta. En la terminología del reporte Foresight (2011) se describe como alguien de gran movilidad y muy emprendedor al momento de forjar su destino bajo condiciones adversas y que participa en la "adaptación transformativa», principio opuesto a la simple «adaptación incremental». En biopolítica, la figura del migrante resiliente (no el concepto de adaptación) podría interpretarse como la reencarnación neoliberal del migrante contemporáneo (Bettini, 2014).

En general, el uso de la resiliencia como una estrategia para adaptarse al cambio climático se vincula de modo estrecho con las principales ideas de la asociación entre migración y desarrollo. Sobre la base de sus propios recursos, los migrantes producen beneficios que se consideran positivos para quienes se quedan en el lugar de origen, en concreto, miembros de la familia, e incluso colectivamente. Lo anterior se parece a la idea de los «migrantes como agentes del desarrollo», en el reciente debate sobre migración y desarrollo, alentado por el Banco Mundial a comienzos de los años 2000. El principal mecanismo que permite la consecución de la «triple-ganancia», tanto para los migrantes y aquellos que les importan (incluyendo sus comunidades de origen), como para los países de emigrantes y los Estados de inmigración, son las remesas financieras y sociales. Esto significa que, en última instancia, son los propios migrantes los responsables de procurar la adaptación al cambio climático, de modo que eximen de responsabilidad a los gobiernos y a las organizaciones internacionales.

Se requiere entonces, como ya se había indicado, de una tercera generación académica que se constituya a partir de la segunda que aún está en curso. En general, la idea de adaptación como agencia proactiva debería conservarse mas no el concepto de resiliencia que es excesivamente ideológico. Se debe tomar en cuenta más que nunca que la adaptación a cambios climáticos adversos (re) produce aspectos socioestructurales y de estratificación social, como las desigualdades sociales. Un hallazgo trascendental y consolidado es que aquellos que carecen de poder, estatus, recursos y quienes, por tanto, están en desventaja relativa con respecto a esos criterios, son los más vulnerables a los efectos negativos del cambio climático (McLeman, Schade y Faist, 2016). Todavía más: la vulnerabilidad y la exposición a las consecuencias del cambio climático es más alta en regiones y comunidades definidas como economías extractivas (materias primas), conectadas con una débil institucionalización de la sociedad civil, en especial ingresos altos y desigualdades de riqueza y un grado relativamente bajo de libertad de prensa (Roberts y Parks, 2007). 
Al considerar el amplio contexto en el que está ocurriendo la migración relacionada con el clima, es relevante notar que los principales productores de los cambios climáticos, cuyos efectos negativos en las oportunidades de la gente, se hallan geográfica y socialmente separados de los más afectados, quienes replican con varios argumentos. Sin embargo, las percepciones sobre esos argumentos están sesgadas. La mayor parte de la atención en los países de la OECD y en la investigación se concentra en grupos capaces de llevar a cabo la migración internacional. En adición, la migración transfronteriza usualmente requiere más recursos que la migración interna o algún tipo de ajuste in situ. Se presta menos atención a las personas que son desplazadas al interior de los estados y a aquellos que no tienen esperanza y han sido despojados, incluso aunque pretendieran alejarse de zonas peligrosas no pueden moverse. Nociones como la vulnerabilidad propician dudas acerca de las capacidades diferenciales para enfrentar los cambios climáticos. A fin de evaluar las desigualdades sociales, se utilizan normas de igualdad, por ejemplo la satisfacción de los derechos humanos básicos, considerados como parámetros que permiten estimar otras categorías de desigualdades. En resumen, esta tercera generación aquí prevista plantea la manera en que los eventos socionaturales se encuentran implicados en la reproducción de las desigualdades sociales y la estratificación actuales, complementariamente contribuye al cambio de las instituciones sociales y de las percepciones culturales en torno a la problemática socionatural.

\section{La reproducción de desigualdades categóricas}

Las desigualdades sociales aluden a la distribución desigual de costos y beneficios referidos a bienes entre unidades sociales tales como individuos, grupos, organizaciones, regiones y estados. Los bienes o recursos involucrados pueden ser de tipo económico (ingresos, propiedad de la tierra); político (autoridad del hogar, autoridad en el lugar de trabajo, autoridad legal); cultural (estilo de vida); social (acceso a redes sociales, es decir, capital social); honorífico (prestigio, reputación); civil (derechos, ciudadanía); y humano (habilidades, educación formal). La distribución desigual de esos recursos involucra diversas posibilidades para acceder $a$ y para moverse entre, por ejemplo, las posiciones sociales definidas por las clases. Propician además, la diferencia en términos de oportunidades de vida, aun así se vislumbra cierta disparidad en cuanto al acceso y 
al empleo de recursos. Las desigualdades no siempre son observables de modo directo, en especial si se toman en cuenta los patrones de desigualdad muy arraigados, como las estructuras de clase (cfr. Van Hear, 2014). En el otro extremo del espectro, varios patrones de desigualdad se observan y miden con mayor facilidad, por ejemplo la movilidad social de individuos y grupos.

Pese a que en ciertas categorías, como el género, la desigualdad no está ausente, es indispensable distinguir de modo analítico entre dos conceptos, es decir, categorías que denotan heterogeneidad pero muestran la diferencia. Heterogeneidad no es lo mismo que desigualdad. Ciertas heterogeneidades - género, etnicidad, edad, estatus legal, religión y grado en el cual las personas o grupos mantienen vínculos a través de las fronteras (transnacionalismo) - en términos de la desigualdad radica en la adscripción de los grupos sociales a las categorías: las distinciones categóricas y las clasificaciones culturales de las heterogeneidades atribuidas a individuos y grupos poseen implicaciones tangibles con relación a la distribución de recursos materiales y simbólicos. Esto supone que las categorizaciones referidas a heterogeneidades derivan en desigualdades sólo si dichas transacciones reproducen límites estables y persistentes entre categorías. De ahí que la expresión «desigualdades categóricas» (Massey, 2007) sea apropiada para describir los procesos de categorización a partir de heterogeneidades. La causalidad entre desigualdad y migración dentro del cambio climático se puede observar en ambos sentidos, desde las desigualdades hacia la migración y viceversa.

Concerniente a la migración como resultado de la desigualdad, ya se ha documentado que la clase como heterogeneidad desempeña un papel fundamental, puesto que los segmentos más pobres de la población son vulnerables a los riesgos medioambientales (para las siguientes afirmaciones empíricas, véase los estudios de caso en McLeman, Schade y Faist, 2016). Estos grupos normalmente pueden moverse dentro de sus países; no obstante, el cruzar las fronteras internacionales a largo plazo podría ser la única opción, tal es el caso de los Estados Isla que se han hundido. En ese contexto, la relocalización puede garantizar una vida digna; todos los habitantes de la isla Kiribati han sido relocalizados (Schade, 2013). Aunque en ocasiones esos costosos programas podrían ser un fracaso, por ejemplo, los inuits de Alaska fueron relocalizados en áreas que en la actualidad se están convirtiendo en pantanos (Bronen, 2013). Referente a las poblaciones atrapadas que ni son capaces de generar una adaptación in situ, ni de optar por la migración como adaptación la situación es precaria. Esta categoría de personas 
constituye la mayor parte de los afectados por la destrucción del medio ambiente y el cambio climático. La intersección entre clase y género representa un nexo obvio: las mujeres son particularmente vulnerables, al igual que los que no tienen tierra, pues la probabilidad de que ellas mueran en el caso de un desastre natural es ocho veces mayor que la de los hombres (Adeniji, 2011; IPCC, 2014).

Las personas que no son pobres deciden migrar como un mecanismo para acumular oportunidades (Tilly, 2005), ello comprende la disponibilidad de una cierta cuantía de recursos financieros o lazos sociales que reduzcan los costos y los riesgos de la migración transfronteriza de larga distancia. Más allá de las heterogeneidades mencionadas (clase, género, edad, religión, localización/ciudadanía o etnicidad), son las espaciales las que marcan la diferencia al momento de enfrentar los riesgos inducidos por el cambio climático. La población en áreas urbanas se caracteriza por su capacidad para manejar el cambio climático, en el Norte global más que en el Sur global, etcétera. La gente suele migrar en la dirección equivocada hacia lugares amenazados por inundaciones y no lejos de ellas. Así sucede con la migración hacia áreas urbanas en los deltas de los ríos de mínima elevación, como Dhaka y Shanghai (Lasailly-Jacob y Peyraut, 2016).

En cuanto al sentido contrario, es decir, de la migración a las desigualdades, las remesas son un planteamiento esencial en la investigación, se argumenta que las remesas financieras (y sociales) de la migración transfronteriza suelen reproducir las estructuras de clase en la población de origen. Existe también un cúmulo de evidencia, por ejemplo, en los Estados Isla del Pacífico y en México, las remesas tienen efectos ambiguos en tanto contribuyen a la reducción de la pobreza y exacerban las desigualdades (véase Aksakal y Schmidt, 2015). En sí, las remesas poseen ventajas y desventajas debido a la ubicuidad de los riesgos de daño moral en la escala de los estados y de los hogares. Los gobiernos emplean las remesas con la intención de corregir los déficits monetarios, incluso podrían evitar cambios estructurales en la provisión de la protección social, como en la salud y la educación. Después de todo, gran parte se destina a esas dos áreas. Con respecto al hogar, los familiares podrían hacerse más dependientes al contar con el ingreso proveniente del exterior en vez de reconstituir las fuentes de ingreso locales (Horst et al., 2014).

El estatus legal es otra heterogeneidad asociada con las desigualdades en la migración. Se trata de algo crucial puesto que se refiere a la constitución políticolegal de la categoría de refugiados climáticos. Uno de los obstáculos científicos fundamentales $-\mathrm{y}$ aquí es donde de nuevo la cuestión socionatural se vuelve 
preponderante - es la dificultad de catalogar legalmente a los migrantes como refugiados en el contexto del cambio climático, lo que haría factible su protección (Kälin, 2015). Es casi imposible asignar con precisión desastres naturales particulares como consecuencia del cambio climático. Pese a que no se puede establecer ninguna causa legal con relación a la causalidad entre cambio climático y migración, existen diversas opciones y por ende un amplio espacio social para los «innovadores de reglas» (Sunstein, 1996). ${ }^{2}$ Este tipo de emprendedores ha tratado activamente de constituir derechos humanos específicos para refugiados en situaciones de migración inducida por el clima. Una de sus propuestas busca implementar una relocalización planificada y es quizá el elemento básico de su propuesta para un régimen de refugiados climáticos (Bierman y Boas, 2008).

Los autores han definido el término «refugiado climático» tomando en cuenta solamente a las personas que huyen de los efectos directos del cambio climático (al interior o a través de las fronteras), es decir, el aumento del nivel del mar, eventos climáticos extremos (sequías y escasez de agua). En ese contexto, el uso del término «refugiado» resultó muy controversial a causa de su significado legal bajo los términos de la Convención de Ginebra para los Refugiados. Asimismo, el Alto Comisionado de la Naciones Unidas para los Refugiados (UNHCR), que es la organización de la Naciones Unidas para los refugiados, rechazó la utilización del término «refugiado climático» o «refugiado medioambiental» y cualquier intento de extender el mandato de la Convención. Se arguyó que «otorgar el estatus de refugiado a los refugiados medioambientales distorsionaría la definición y haría presión sobre los extremadamente escasos recursos del régimen internacional para los refugiados» (Suhrke, 1994:492). Dicha oposición podría ser una de las razones por las cuales los términos «migración medioambiental» $\mathrm{y}$ «migración climática» predominaron desde entonces en el debate y la investigación. Es notoria la existencia de argumentos adicionales en el debate acerca de la necesidad de códigos legales para los migrantes inducidos por el cambio climático.

${ }^{2}$ Nota de los traductores: se ha traducido libremente la expresión original «norm entrepeneurs» haciendo énfasis en la connotación innovadora y creativa de los empresarios y emprendedores. 


\section{Perspectiva: inclusión de la problemática socionatural}

Con antelación se mencionó que la primera generación de estudiosos del cambio climático y la migración, al usar un enfoque mecánico de la "naturaleza», subestimaron las capacidades adaptativas de los seres humanos frente a los incipientes cambios ecológicos. La segunda generación de académicos se concentró en un tipo particular de agencia con respecto a la «sociedad». El principal protagonista fue el migrante resiliente que procura adaptarse exitosamente al cambio climático. Esta nueva generación difundió una versión muy neoliberal de la movilidad: un migrante móvil y dócil que actúa de manera anticipada y previsora. Complementariamente, no se ha prestado suficiente atención al hecho de que no se puede probar la relación entre el cambio climático y los eventos particulares, al menos no por medio de los métodos de las ciencias naturales. Al asumir un enfoque combinado «naturaleza-sociedad» (cfr. Mooney, Duraiappah y Larigauderie, 2011), nos percatamos que la migración mantiene intactas las profundas estructuras de las desigualdades sociales y refuerza mecanismos de exclusión (cfr. Faist, 2016). Este obstáculo no ha impedido a los innovadores de reglas denunciar el terrible destino de muchos migrantes que aceptan o incluso se hallan forzados a la movilidad inducida por el cambio climático.

La investigación debe ampliarse y no sólo vincular el cambio climático con las desigualdades, sino además incorporar la violencia civil. En la mayoría de los casos, el cambio climático y la violencia son considerados como dos amenazas independientes, cada una contribuye de manera potencial al flujo de migrantes en el mundo. Estudios recientes indican que el cambio climático y la violencia civil se interrelacionan causalmente. Se sabe que los estallidos de violencia civil se asocian de forma estrecha con el fenómeno de El Niño - Oscilación Sur(ENOS, por sus siglas en inglés) (Hsiang, Meng y Crane, 2011). La probabilidad de que surja un conflicto civil durante los años de El Niño se duplica con relación a La Niña; todavía más, el ENOS podría explicar el 21 por ciento de los conflictos civiles entre 1950 y 2005. Se trata de una correlación muy significativa, ellos aunado al hecho de la evidencia que sugiere un nexo entre el calentamiento global y un mayor riesgo de violencia civil en gran parte del África Subsahariana (Burke et al., 2009). Dado el potencial del cambio climático para influir en la frecuencia y severidad de eventos climáticos como El Niño, no solamente el calentamiento global tiene la capacidad de generar migración directamente a través del desplazamiento, lo hace de igual modo indirectamente al 
desencadenar conflictos civiles en las áreas afectadas en el mundo. Otra vez las guerras civiles producen más migración y flujos de refugiados. Sin embargo, se conoce muy poco sobre los mecanismos sociales involucrados.

Referente a la transformación en las percepciones del cambio climático, la migración debe situarse en el contexto de las transformaciones político-económicas generales, la más característica es el modo de organización de la vida económica. Algunos expertos aluden a una «fisura metabólica» (Foster, 1999). Este término se refiere a las tendencias hacia las crisis ecológicas en el marco del capitalismo. Karl Marx ya había teorizado una ruptura en la interacción metabólica entre la naturaleza y la sociedad/cultura derivada del modo de producción capitalista y la creciente brecha entre las zonas urbanas y rurales. Hizo hincapié en una «grieta irreparable en el proceso interdependiente del metabolismo social» (Marx, 1981:949). Sostuvo incluso, que esa brecha era irreconciliable con cualquier tipo de sustentabilidad (cfr. Rosa et al., 2015) y con la explotación de los hombres comparable a la explotación del suelo. Paralelamente, otro de los fundadores de la sociología, Max Weber, declaró que la sociedad industrial funcionaría "hasta que la última tonelada de combustible fósil fuese reducida a cenizas». Se ha demostrado que el capitalismo se mantiene como una fuerza universal y al mismo tiempo «local en todas partes» (Latour, 1993:117). Es justo en la escala local donde han tenido lugar los conflictos sobre mitigación y adaptación al cambio climático en los últimos años, lejos de reuniones mundiales espectaculares pero inconsecuentes. Son los conflictos climáticos locales y no la gobernanza climática global los que han logrado algún progreso en el tratamiento del rampante incremento del carbono en la atmósfera.

\section{Referencias}

Adeniji, Grace (marzo de 2011), "Adapting to climate change in Africa», Jotoafrika (6), pp. 1-8. Ahmed, Ishtiaq (1997), «Exit, voice and citizenship», en Tomas Hammar, Grete Brochmann, Kristof Tamas y Thomas Faist (eds.), International migration, immobility and development. Multidisciplinary perspectives, Oxford, Berg, pp. 159-186.

Aksakal, Mustafa y Kerstin Schmidt (2015), «Migration and social protection as adaptation in response to climate-related stressors: the case of Zacatecas in Mexico», en Felicitas Hillmann, Marie Pahl, Birte Rafflenbeuly Harald Sterly (eds.), Environmental change, adaptation and migration: bringing in the region, London, Palgrave Macmillan. 
Bettini, Giovanni (2014), "Climate migration as an adaption strategy: de-securitizing climate-induced migration or making the unruly governable?», Critical Studies on Security, 2(2), pp. 180-195.

Bierman, Frank e Ingrid Boas (noviembre-diciembre de 2008), "Protecting climate refugees: the case for a global protocol», Environment: Science and Policy for Sustainable Development, pp. 9-16, in http://www.environmentmagazine.org/Archives/ Back\%20Issues/November-December\%202008/Biermann-Boas-full.html

Black, Richard, Dominic Kniveton y Kerstin Schmidt-Verkerk (2013), «Migration and climate change: toward an integrated assessment of sensitivity», en Thomas Faist y Jeanette Schade (eds.), Disentangling migration and climate change, Dordrecht, Springer, pp. 29-54.

Bronen, Robin (2013), Climate-induced displacement of Alaska native communities, Alaskan immigration justice project, Alaska, Alaska Immigration Justice Project/Brookings-LSE/Project on Internal Displacement.

Burke, Marshall B., Edward Miguel, Satyanath Shanker, John A. Dykema y David B. Lobell (2009), "Warming increases the risk of civil war in Africa», Proceedings of the National Academy of Sciences, 106(49), pp. 20670-20674.

Castles, Stephen (2007), "Twenty-first-century migration as a challenge to sociology», Journal of Ethnic and Migration Studies, 33(3), pp. 351-371.

Dunlap, Riley E. y William R. Catton (1979), "Environmental sociology», Annual Review of Sociology, 5, pp. 243-273.

Durkheim, Émile (1964), The rules of sociological method, New York, Free Press.

El-Hinnawi, Essam (1985), Environmental refugees, Nairobi, United Nations Environmental Programme (UNEP).

Ellis, Frank (2000), Rural livelihood diversity in developing countries: analysis, policy, methods, Oxford, Oxford University Press.

Etzold, Benjamin, Ahsan Uddin Ahmed, Selim Reza Hassan, Sharmind Neelormi y Tamer Afifi (2016), "Rainfall variability, hunger, and social inequality, and their relative influences on migration: evidence from Bangladesh», en Jeanette Schade y Thomas Faist (eds.), Environmental degradation and social inequalities, Dordrecht, Springer, pp. 27-42.

Faist, Thomas (2009), «The transnational social question: social rights and citizenship in a global context», International Sociology 24(1), pp. 7-35.

(2016), "Cross-border migration and social inequalities», Annual Review of Sociology, 42, pp. 323-346. 
Faist, Thomasy Jeanette Schade (2013), "The climate-migration nexus. A reorientation», en Thomas Faist y Jeanette Schade (eds.), Disentangling migration and climate change: toward an analysis of methodologies, political discourses and human rights, Springer, Dordrecht, pp. 3-25.

Foresight (2011), Foresight: migration and global environmental challenge. Final project report, London, The Government Office for Science.

Fornalé, Elisa, Jeremie Guélat y Etienne Piguet (2016), «Framing labour mobility options in small island States affected by environmental changes», en Robert McLeman, Jeanette Schade y Thomas Faist (eds.), Environmental degradation and social inequalities, Dordrecht, Springer, pp. 167-188.

Foster, John Bellamy (1999), "Marx's theory of metabolic rift: classical foundations for environmental sociology», American Journal of Sociology, 105(2), pp. 366-405. (2000), Marx's ecology: materialism and nature, New York, Monthly Review Press.

Giddens, Anthony (2009), The politics of climate change, Cambridge, Polity.

Horst, Cindy, Marta Bivand Erdal, Jorgen Carling y Karin Afeef (2014), «Private money, public scrutiny? Contrasting perspectives on remittances», Global Networks, 14(4), pp. 514-532.

Hsiang, Solomon M., Kyle C. Meng y Mark A. Crane (2011), "Civil conflicts are associated with the global climate», Nature, 476, pp. 438-441.

Human Development Report (2007), Fighting climate change: human solidarity in a divided world, New York, United Nations Development Program (UNDP).

Intergovernmental Panel on Climate Change (IPCC) (2014), Climate change 2014: synthesis report. Contribution of working groups I, II and III to the fifth assessment report of the Intergovernmental Panel on Climate Change, Geneva, IPCC.

Kälin, Walter (noviembre de 2015), «Klimaflüchtlinge, katastrophenvertriebene oder schutzlose migranten? Flucht in Zeiten des klimawandels», VHS-Bulletin (4), pp. 10-18.

Lassailly-Jacob, Véronique y Malika Peyraut (2016), «Social and spatial inequality linked to flood-induced displacements in Burkina Faso in 2009 and 2010», en Robert McLeman, Jeanette Schade y Thomas Faist (eds.), Environmental degradation and social inequalities, Dordrecht, Springer, pp. 57-72.

Latour, Bruno (1993), We have never been modern, Cambridge, Harvard University Press. Marx, Karl (1981), El capital, vol. 3, London, Penguin.

Massey, Douglas S. (1990), «Social structure, household strategies, and the cumulative causation of migration", Population Index, 56(1), pp. 3-26. 
(2007), Categorically unequal: the American stratification system, New York, Russell Sage Foundation.

McLeman, Robert, Jeanette Schade y Thomas Faist (2016), Environmental degradation and social inequalities, Dordrecht, Springer.

Mooney, Harold A., Anantha Duraiappah y Anne Larigauderie (2011), «Evolution of natural and social science interactions in global change research programs", PNAS, 10(1), pp. 3665-3672.

Myers, Norman y Jennifer Kent (1995), Environmental exodus, an emergent crisis in the global arena, Washington DC, Climate Institute.

Poirine, Bernard (1997), "A theory of remittances as an implicit family loan arrangement», World Development, 25(4), pp. 589-611.

Roberts, J. Timmons y Bradley C. Parks (2007), A climate of injustice: global inequality, North-South politics, and climate policy, Cambridge, MIT Press.

Rosa, Eugene A. et al. (2015), "The human (anthropogenic) driving forces of global climate change», en Riley E. Dunlap y Robert J. Brulle (eds.), Climate change and society: sociological perspectives, New York, Oxford University Press.

Schade, Jeanette (2013), "Climate change and planned relocation: risks and a proposal for safeguards», en Thomas Faist y Jeanette Schade (eds.), Disentangling migration and climate change, Dordrecht, Springer, pp. 183-206.

Shen, Shawn y François Gemenne (2011), «Contrasted views on environmental change and migration: the case of Tuvaluan migration to New Zealand», International Migration, 49(S1), pp. e224-e242.

Simonet, Guillaume (2010), "The concept of adaptation: interdisciplinary scope and involvement in climate change», Sapiens, 3(1), en https://sapiens.revues.org/997

Suhrke, Astri (1994), "Environmental degradation and population flows», Journal of International Affairs, 47(4), pp. 473-496.

Sunstein, Cass R. (1996), "Social norms and social roles», Columbia Law Review, 96(4), pp. 903-968.

United Nations Development Program (UNDP) (2007), Human development report 2007/2008. Fighting climate change. Human solidarity in a divided world, New York, Palgrave.

United Nations Framework Convention on Climate Change (UNFCCC) (1992), United Nations framework convention on climate change, Bonn, UNFCCC.

Van Hear, Nicholas (2014), «Reconsidering migration and class», International Migration Review 48(S1), pp. S100-S121. 